\title{
The use of modern measurement techniques for designing pro ecological constructions
}

\author{
Michał Wieczorowski ${ }^{1{ }^{*},}$, Bartosz Gapiński ${ }^{1}$, Maciej Szymański ${ }^{2}$, and Artur Rękas ${ }^{3}$ \\ ${ }^{1}$ Poznan University of Technology, Institute of Mechanical Technology, Piotrowo 3, 60-965 Poznan, Poland \\ ${ }^{2}$ Ster Sp. z o.o., Czluchowska 12, 60-434 Poznan, Poland \\ ${ }^{3}$ AGH University of Science and Technology, Faculty of Non-Ferrous Metals, Mickiewicza 30, 30-059 Krakow, Poland
}

\begin{abstract}
In the paper some possibilities of application modern length and angle metrology techniques to design constructions that support ecology were presented. The paper is based on a project where a lighter bus and train car seat was developed. Different options were presented including static and dynamic photogrammetry, computed tomography and thermography. Research related with dynamic behaviour of designed structures gave input to determine deformation of a seat and passengers sitting on it during communication accidents. Works connected to strength of construction elements made it possible to optimize its dimensions maintaining proper durability. Metrological actions taken in relation to production machines and equipment enabled to better recognize phenomena that take place during manufacturing process and to correct its parameters, what in turns also contributed to slim down the construction.
\end{abstract}

\section{Introduction}

Contemporary constructions are required not only to fulfil functional parameters and assure utility features on a certain level of aesthetics but also to support pro ecological activities [1]. One of the directions connected with such a thinking is designing light solutions, still maintaining all the necessary strength parameters. It is particularly visible when various parts of vehicle equipment is concerned, where every reduced kilogram saves fuel and reduces emission of polluting substances to the atmosphere.

In the paper these works were discussed on an example of a seat manufactured by company STER, who is a leading and renowned in the world supplier of that kind of products to many leading bus (for different purposes) and train car manufacturers. The aim of a project realized by a company and research institutions was then elaboration of a seat with a mass significantly smaller than the versions manufactured so far. As an effect reduction of nearly $10 \mathrm{~kg}$ was achieved, what makes about $30 \%$ of total mass of a seat and - as an example - gives a $400 \mathrm{~kg}$ less total weight of an average bus. That in turns reduces fuel consumption even up to as much as 21 per $100 \mathrm{~km}$.

For research and inspection purposes advanced metrology solutions and special constructions were applied, helping to visualize on-going processes, verification of placed research hypotheses obtaining data for simulation purposes with very low measurement uncertainty. This topic is not widely discussed in references, the manufacturers of bus seats and structures have to comply with different formal regulations, regardless how this is achieved or measured.

Technological progress in XX century enabled development of coordinate measurement technique. Thus, it is possible to obtain information regarding shape and dimensions of an inspected workpiece basing on a set of measurement points described by means of coordinates in space. These data are transformed by measurement software into related elements that are a reference for designation of dimensions and deviations of a measured part in accordance with its geometrical product specification (GPS). A measurement of free form surfaces and their assessment in relation to a CAD model is also possible. Coordinate technique facilitates these tasks to a great extent or even makes them possible to happen or execute. Without it e.g. determination of many position deviations would not be possible, just because of difficulty in definition centre of a circle (Fig. 1).

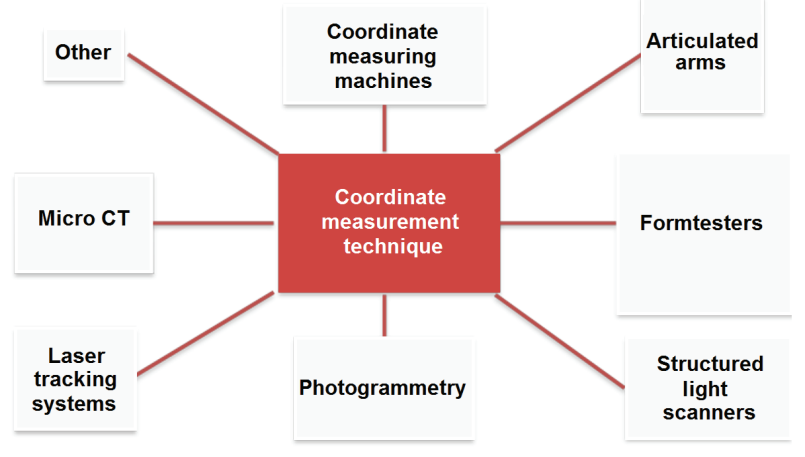

Fig. 1. Application of coordinate measurement technique in measurement devices [2].

\footnotetext{
* Corresponding author: michal.wieczorowski@put.poznan.pl
} 
That is why we have a bunch of application possibilities of coordinate measurement technique in different devices, what was in details depicted on Fig. 1. This graph presents a general division of measurement instruments in which analysis of points coordinates on measurement surfaces was used to determine geometrical specification of an element. Such a differentiation of measurement instruments has its source in complexity of measurement tasks and tendency to obtain as versatile measurement information as possible, with reliable credibility and including economic aspect. These devices have then different measurement methods, number and way of collecting points and accuracy parameters and these features significantly contribute to decision which of them should be chosen in particular metrology task.

\section{Static and dynamic photogrammetry}

Seats for public transportation must ensure specific needs depending on their designation and function. Separate set of requirements features the ones that are manufactured for tourist buses equipped with seat belts, another one for city buses and still another one for train cars. Still, in each case, though to a different extent, it is necessary to fulfil strength requirements regarding static and dynamic deformation. When inspecting both: single elements and whole construction sets of seats it is necessary to use photogrammetry techniques. Accuracy parameters of these systems assure metrologically correct measurement results, while their flexibility makes it possible to map even very complicated free form surfaces. Photogrammetry itself can be defined as a method to obtain three dimensional data about shape and location of objects basing on one or many two dimensional images $[3,4,5]$. In length and angle metrology while measuring geometric features of workpieces, photogrammetry means measurement of clearly coded characteristic elements on objects. They are usually markers: points, lines or fringes projected onto the object by a projector. Photogrammetric systems used in measurement techniques allow for digitization of a scanned object. There are two basic groups of methods: static and dynamic ones. In relation to this project both of them were used. To inspect complex elements as seats for public transportation it is necessary to verify geometry of single pieces and the whole structure. This allows to assess manufacturing process and final assembly. Knowledge about real shape of a seat gives opportunity to confirm its dimensional compatibility with specification. It is also a necessary condition to ensure possibility of applying a certain seat to static and dynamic deformation tests.

To assess seat geometry as well as effects of research a scanning system with structural light was used. It was composed of a projecting light source and two CCD cameras registering layout of projected fringes on the object surface. Working principle of this device is based on triangulation [6], what was shown on Fig. 2. Basing on optical transformation equations a processing unit calculates 3D coordinates for each camera pixel. A result of such an action is a cloud including up to several million points for each single measurement shot.
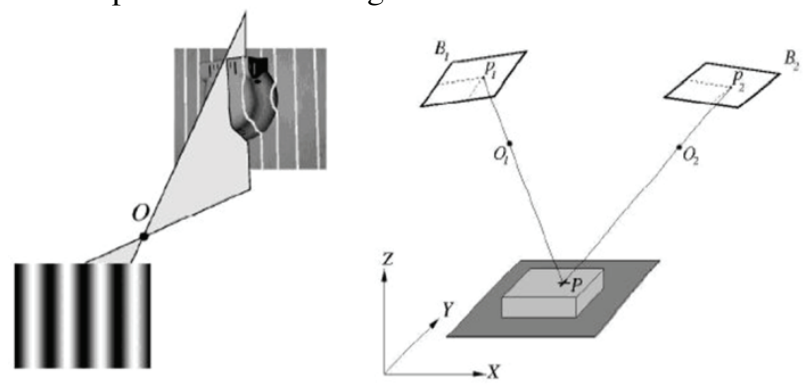

Fig. 2. Projection of fringes and triangulation.

A projecting system and cameras can be integrated into one sensor head. Thanks to triangulation it is possible to compare real data with nominal ones and workpieces before and after strength tests. This allows for estimation of plastic deformations of particular parts of seat structure and deformation of the whole geometry in relation to mounting places. An example of a seat with a list of measurement points and dimensional deviations was shown on Fig. 3.

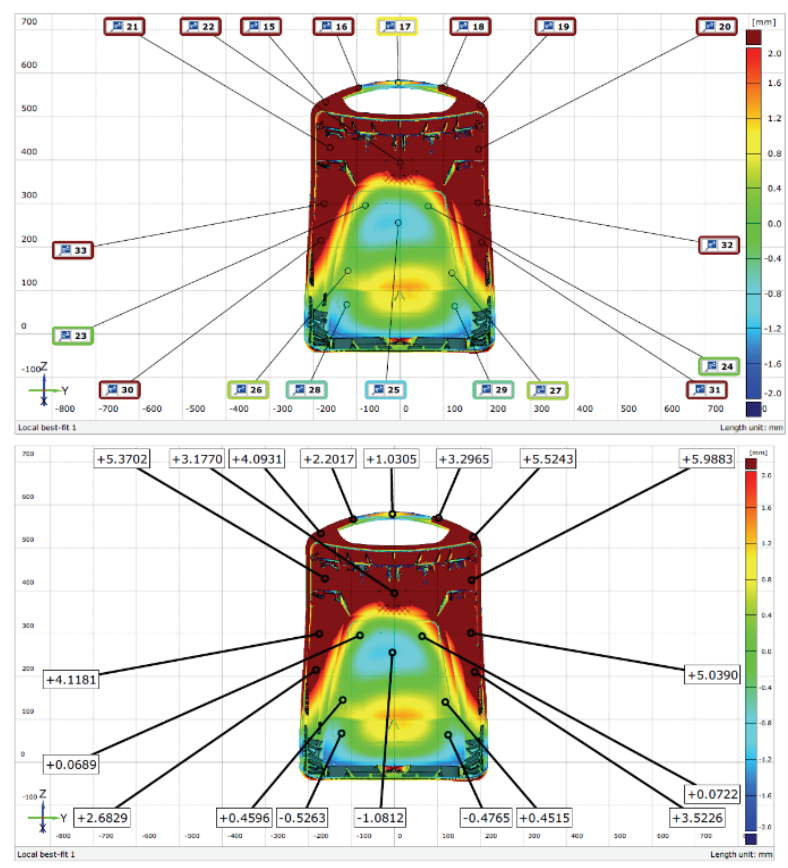

Fig. 3. A photogrammetric image of a bus seat with color map of deviations.

Thanks to these measurements seat sections were modified and reinforcement areas were defined, as well as a cooling method was elaborated that would not cause significant dimensional changes.

In dynamic measurements, fast cameras register displacements of point located on measured object. Its full dynamic behavior is obtained from many images recorded with great frequency. Because of a great number of measurement points a system enables to get a precise analysis of deformation. The first presented test shows analysis of closing process of a mould with different closing forces. An injection moulding machine had different possibilities from 500 to $1200 \mathrm{kN}$, so 
measurements were taken in $100 \mathrm{kN}$ intervals. For all measurements reports were made with identically defined coordinate system. Points were analyzed in $\mathrm{X}$ axis, i.e. in mould closing direction. A measurement result for $1200 \mathrm{kN}$ closing force is shown on Fig. 4 .
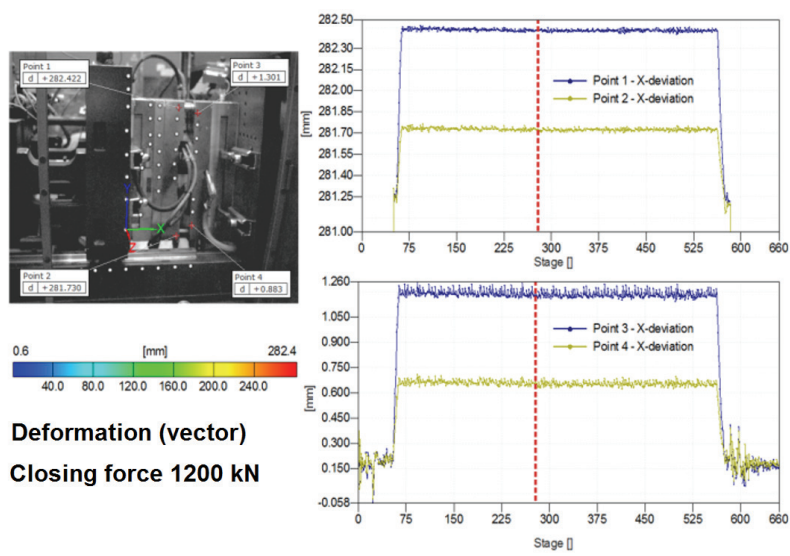

Fig. 4. Closing force measurement report for $1200 \mathrm{kN}$, X axis.

Basing on this procedure (together with thermography research shown in chapter 3) it was possible to determine proper working parameters of injection moulding machine to obtain a workpiece with expected geometry.

Dynamic photogrammetry was also used to analyze seat behavior during static and dynamic tests connected with its strength. To ensure proper dynamics of a measurement displacement of special appropriately located markers is considered. It also allows to calculate velocity and acceleration. All this analysis was performed in relation to independent reference points as well as relatively e.g. for lower part of seat and support. In research a measurement space in a form of cube with $2 \mathrm{~m}$ side was used [7]. Such a configuration enables for maintaining proper accuracy parameters (particular points were identified with uncertainty smaller than 0,05 $\mathrm{mm}$ ) and including the whole area of interest where investigated phenomenon takes place. It is particularly important as tests with static and dynamic loads are single ones and their repetition for the same seat is not possible. In static tests a force is applied to a stationary seat while in dynamic ones a seat with mannequins is accelerated to a certain speed and than rapidly stopped to imitate an accident. In both cases a measurement takes place during the test, so - regardless the name - both, static and dynamic measurement really has a dynamic factor.

Dynamic measurements were performed on a specially constructed research track enabling for a full crash test. It allows for accelerating a carriage to 50 $\mathrm{km} / \mathrm{h}$, though a maximum speed of $32 \mathrm{~km} / \mathrm{h}$ was used to fulfil directives and regulations. These tests are made to verify, whether a sitting person is properly kept by a seat belt and a seat before.

As an effect it is possible to determine what kind of injuries can happen to a passenger when accident takes place and if a seat construction with mounting system is strong enough.

On Fig. 5 mannequins during a dynamic test can be observed. Their displacement to the front was blocked on a knee level after a contact with the seat before. In such a situation knees work like hinges and the mannequin body rotates to the front in relation to an axis going through both knees. It effects in a dynamic contact of a head with a seat construction. Because of this, a seat before is plastically deformed.

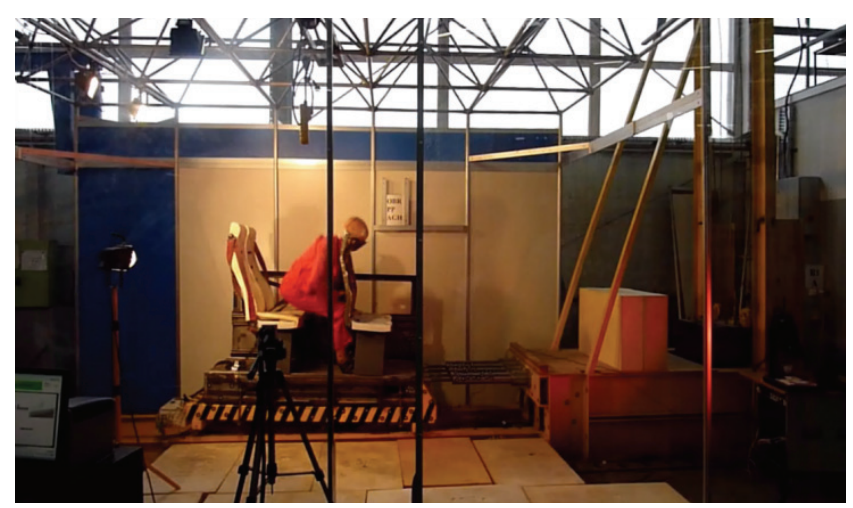

Fig. 5. Heads of mannequins strike seats before.

Fig. 6 shows the first stage of deformation analysis of a seat on the level of bolster after being hit by a mannequin not fastened by a seat belt. Two point are analyzed - one at each bolster.

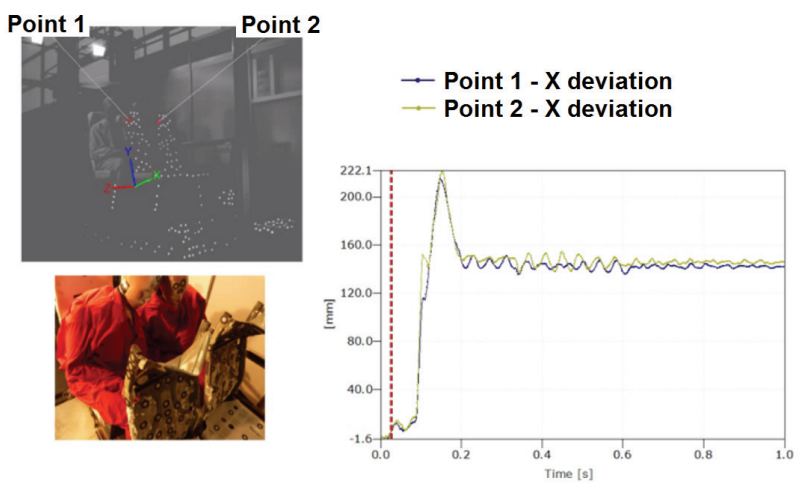

Fig. 6. Bolster and seat deformation after being hit by a mannequin not fastened by a seat belt.

As it was mentioned before, in static tests a force is applied to a stationary seat. This test is executed to determine whether a person is properly kept by a seat and seat belts and would not be seriously injured and whether a seat and its mounting are strong enough. The test has a positive result when a after applying a force a seat would not be elastically deformed more than a certain limit and if after releasing a force plastic deformation will appear as a crash zone. This zone has to take a part of collision energy from a passenger saving his life. Furthermore, seat elements can not be separated and the seat itself must remain stiffly mounted. After such a test there can be no cracks and sharp edges on any seat part. They could cause serious injuries and wounds.

On Fig. 7 a moment is depicted when a swingle on a specially designed research setup striking a seat causes its maximum deflection and deformation. Maximum 
deformation value in highest point of support reaches nearly $29 \mathrm{~mm}$. Value of deviation goes successively down in the seat direction. It is a proper reaction concluding from a fact that the closer is the swingle from the seat the smaller is the arm force on which it operates.

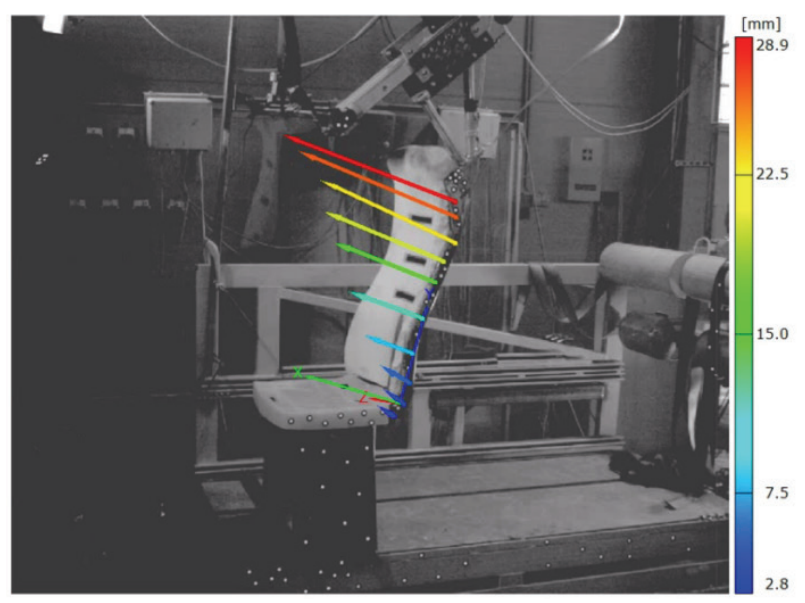

Fig. 7. A research setup with a swingle designed for energy absorption test - a moment of maximum inclination of a seat support.

On Fig. 8 a plot showing change of location of a marker in upper part of support was shown. This place is the most exposed to deformation.

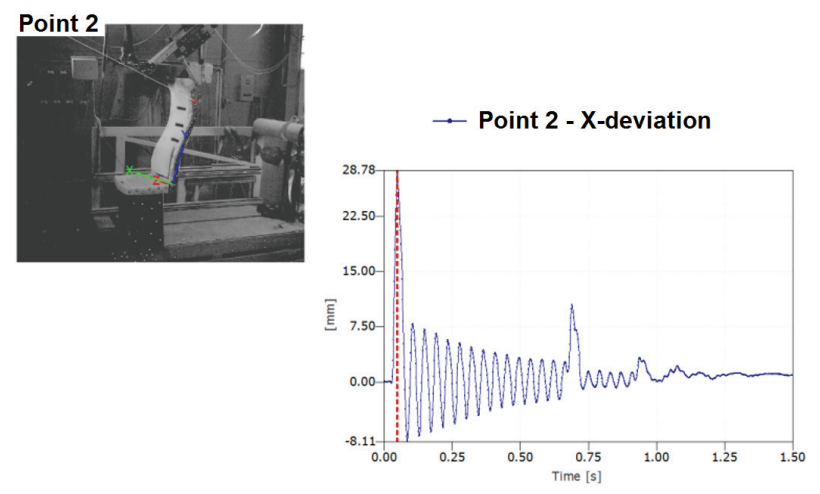

Fig. 8. A research setup with a swingle designed for energy absorption test - a plot showing displacement of a point located in upper part of support.

Analysis of that plot very precisely shows a course of energy absorption test. As it was shown in the moment of strike of a swingle its upper part moves at as much as $29 \mathrm{~mm}$ to the front. Elastic deformation causes push back of a swingle and a support reaction causing its deflection in the opposite direction of $8 \mathrm{~mm}$ from initial position. So the support covered a distance of $37 \mathrm{~mm}$. From that moment it makes a wobbly movement with fading amplitude until a swingle strikes again. Then a deformation is much smaller i.e. about $8 \mathrm{~mm}$. Again it causes a support reaction caused by elastic deformation. A support comes back to the initial position making a wobbly movement with a small amplitude. After 1.25 seconds from a first strike of a swingle a return of a support to initial position can be observed, and deformation value does not exceed $1 \mathrm{~mm}$. This proves a proper construction of a seat, because during test it had nearly only elastic deformation.

Another application of dynamic photogrammetry was testing strength of elements of seat structure. In the research load and deformation parameters from a tensile strength machine were recorder and simultaneously data from photogrammetric system were analyzed. It was necessary to paint the whole sample with white paint and successively put irregular black dots on it to make this kind of measurements. This kind of marking is generally similar to markers in static or dynamic photogrammetry described in chapter two e.g. for geometry inspection. However, for strength tests traditional markers would not allow to get such a versatile information as it is when white paint and black dots are considered. An image of a sample after bending (total deformation) and analysis of changes that took place inside it were shown on Fig. 9.

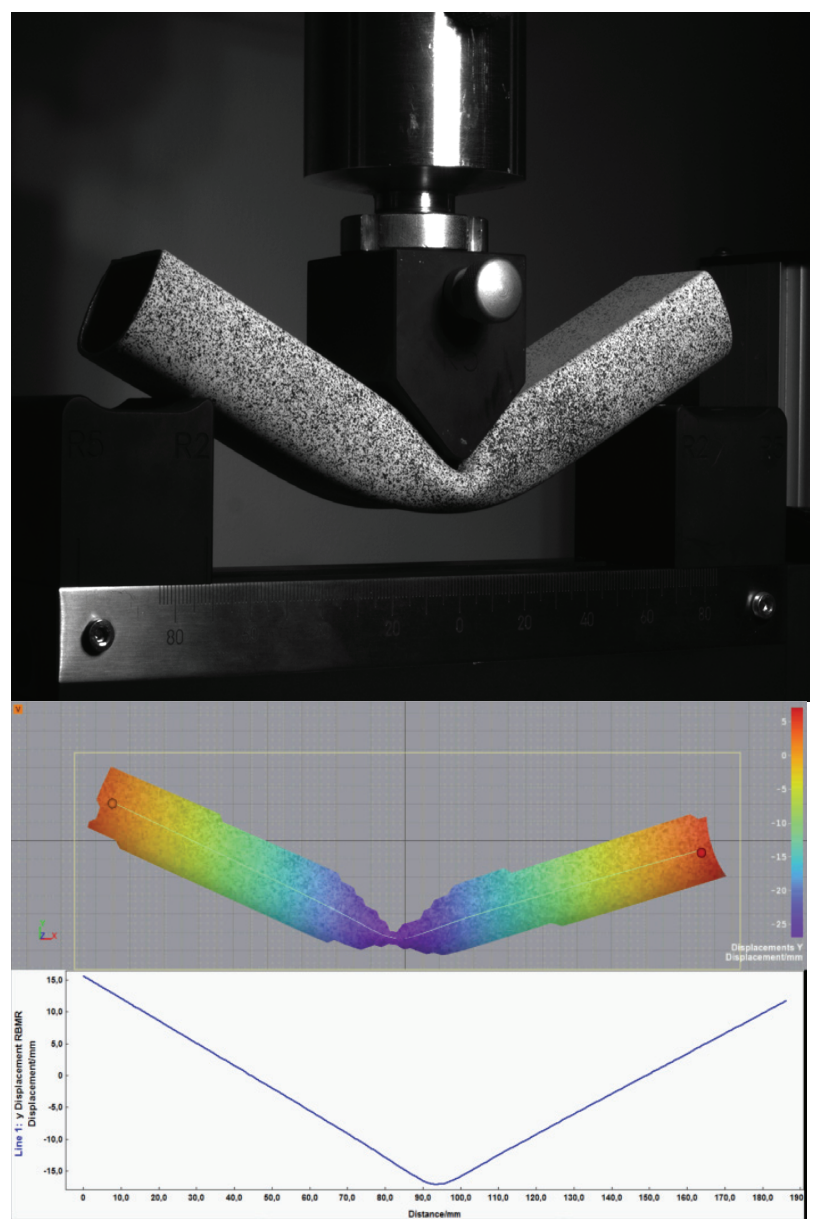

Fig. 9. A sample after total deformation and image of displacements.

Basing on this analysis possibilities of load transfer of a chosen profile was assessed.

\section{Thermography}

A basic feature of thermography systems is the one that they indirectly measure temperature of an inspected workpiece; what is measured directly is a power of infrared radiation emitted by an object. This radiation is 
transferred by a detecting structure of a measurement system into electric signal that carries information about temperature of a part. Temperature in turns is determined with the use of thermometric characteristics of a thermograph written in the memory of a specialized computer designated among others to control process of reproduction of temperature fields seen by a camera of a thermovision system. Apart from radiation emitted by the object (this radiation carries information about its temperature) another radiations gets to a detection structure of a thermograph: reflected from an object and own radiation of atmosphere generated on the segment of path between object and camera. Furthermore, radiation emitted by interior of the camera falls on the detector as well. Each of these above mentioned components of radiation has a different distribution of radial power spectrum density, that depends among others on conditions of atmosphere (humidity, pressure, carbon oxide contents, fog, pollination, emissive properties of an object, emissive properties of camera elements etc.). All this makes that reproduction of object temperature is realized as an effect of computation procedures, including influence that these factors have on useful measurement signal.

A research stand for seat thermal diagnosis was made basing on thermovision camera. What was investigated was the influence of different cooling systems on a ready plastic made seat. An example of a seat during cooling after molding injection is shown on Fig. 10.

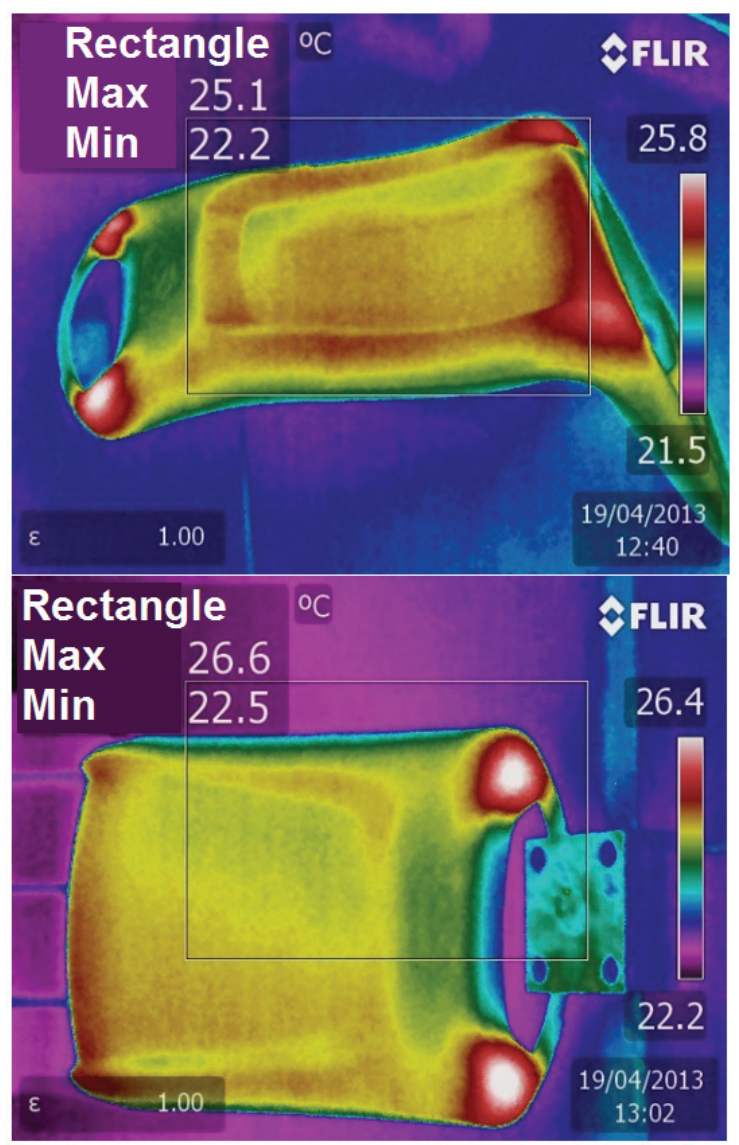

Fig. 10. Images of a seat during a cooling process.
Basing on that kind of thermovision research (including dynamic photogrammetry described in chapter 2) it was possible to optimize injection molding process and cooling method. This in turns dramatically improved repeatability of dimensions and geometrical deviations of a ready seat.

\section{Computed tomography}

Traditional optical or tactile measurement methods quite often suffer from lack of access to places where taking measurements or collecting points is necessary. This can be even more difficult for some of coordinate techniques. Measuring some of the features may require cutting a workpiece which from one side is not effective and from the other one this process itself causes modifications of both: an edge and layers located beneath the surface. This in turns significantly influences on measurement accuracy parameters. A good solution of this problem is using a computed tomograph (CT). This method of measurement has recently allowed to extend measuring possibilities [8]. It applies X-rays going through the object and giving an image on a detector. CT scanners are known in medical applications for a long time, but for technical imaging of 3D objects and extracting geometrical features, these devices are still a kind of novel solution. The image from CT scanner allows for estimation of the geometry of manufactured product as well as internal closed surfaces. It also allows for analysis of pores inside material or estimation of deformation $[9,10]$. Voxel is a unit of 3D spatial image (volumetric element) - and it corresponds to pixel for 2D images. Voxel can be then treated as workpiece diameter divided by a number of pixels. A picture of a tomograph with its working principle was depicted on Fig. 11.

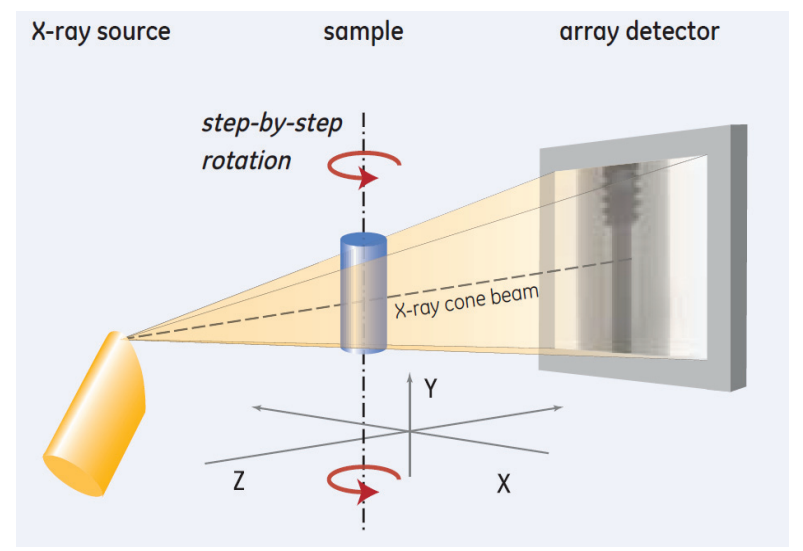

Fig. 11. A CT scanner and its functioning principle.

Preparing tomographic image consists of measurement of radiation absorption penetrating the object. Its volume is divided into voxels which have the same linear coefficient of radiation absorption. The reconstructed sectional image is a quantitative map of the linear coefficient of radiation absorption in voxels which are a part of the scanned slice $[11,12,13]$. The obtained cloud of points allows to conduct the analysis of dimensions and internal structure. 
Computed tomography in the research concerning bus and train car seats was used to analysis of a bended profile [14]. A change in thickness of walls on inner and outer side of arc (being a result of bending) can be observed (Fig. 12). What is understood a wall on the outside surface gets thinner. As it is visible from the drawing a change of thickness is uniform on the whole length of the arc and there are no important local changes, which of course means a positive assessment of the process.

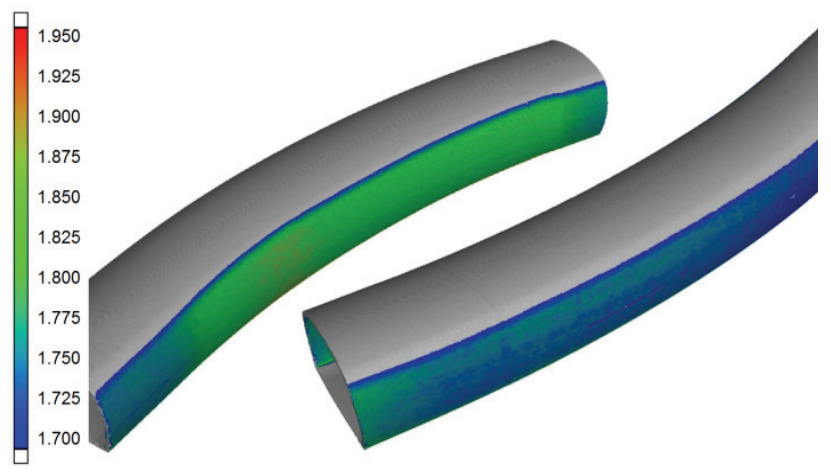

Fig. 12. Wall thickness measurement in the bending area - a view from inner and outer side.

Wall thickness measurement particularly in hardly accessible places is a very good task for computed tomographs. As a result of CT measurements of seat elements a 3D model as a result of inspection was obtained. Basing on that geometry assessment in relation to nominal CAD model is possible (Fig. 13).

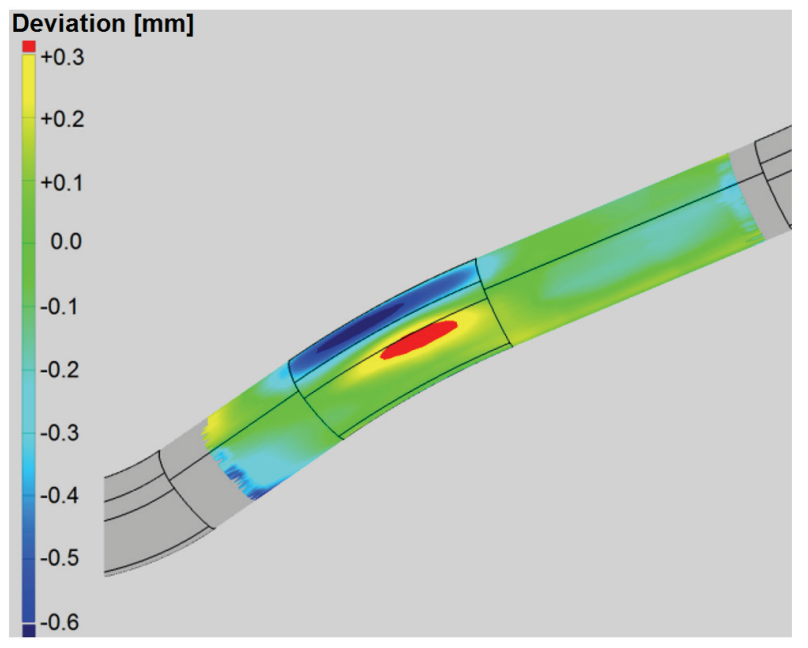

Fig. 13. A map of deviations of a bended profile from nominal CAD model obtained by means of a CT.

These measurements and research made it possible to obtain results that were a base to assess a degree of necking of a wall section. It was also a feedback to design of a profile that would fulfil all the dimensional and strength requirements.

\section{Conclusions}

Modern techniques of length and angle metrology used to aid construction of a new bus seat helped to create lighter solutions, but maintaining required strength parameters. Research related with dynamic behavior of designed structures gave input to determine deformation of a seat and passengers sitting on it during communication accidents. Works connected to strength of construction elements made it possible to optimize its dimensions maintaining proper durability. Metrological actions taken in relation to production machines and equipment enabled to better recognize phenomena that take place during manufacturing process and to correct its parameters, what in turns also contributed to slim down the construction. The paper shows that nowadays designing pro ecological construction requires a lot of metrological actions to prove their functionality.

Part of this work was supported by the Polish National Centre of Research and Development (project contract No. Innotech In-Tech K2/IN2/58/182896/NCBR/12; "Elaboration of manufacturing technology of new generation ultralight seats for public transportation fulfilling requirements of UE directives, UN regulations and American White Book").

\section{References}

1. G.M. Królczyk, Niesłony P. Maruda R.W., Wojciechowski S., J. Cleaner Prod., 142 (2017)

2. L. Marciniak - Podsadna, Methodics of accuracy testing of optical coordinate scanners, $\mathrm{PhD}$ dissertation, Poznań University of Technology, 2014

3. R.J. Campbell, P.J. Flynn, Comput Vision Image Understanding, 81, 2 (2001)

4. D. Caspi, et al., IEEE T Pattern Anal, 20, 5 (1998)

5. R. Majchrowski, Grzelka M., Wieczorowski M., Sadowski L., Gapiński B., Metrology \& Meas Syst, XXII, 4 (2015)

6. M. Wieczorowski, M. Ruciński, R. Koteras, Arch Foundry Eng, 10 (2010)

7. M. Wieczorowski, Gapiński B., Szymański M., Grzelka M., Rękas A., Szymański S., Mechanik, 11 (2016)

8. B. Gapiński, Wieczorowski M., MarciniakPodsadna L., Dybała B., Ziółkowski G., Procedia Eng, 69 (2014)

9. J.P. Kruth, Bartscher M., Carmignato S., Schmitt R., De Chiffre L., Weckenmann A., CIRP Annals, 60 (2011)

10. S. Carmignato, E. Savio, CIRP Annals, 60 (2011)

11. E. Ratajczyk, Mechanik, 2 (2011)

12. A.G. Ramm, Inverse Problems, Tomography and Image Processing, Springer, 1998

13. N. Sorokin, High-Performance Reconstruction in Computer Tomography: Formal Specification and Implementation, VDM Verlag, 2008

14. Gapiński B., Wieczorowski M., Szymański M., Szymański S., Grzelka M., Rękas A., Mechanik, 11 (2016) 